\title{
An optimal fractional LQR-based control approach applied to a cart-pendulum system
}

\author{
Julio Cesar Basilio, José Geraldo Telles Ribeiro, \\ Americo Cunha Jr, and Tiago Roux Oliveira \\ Rio de Janeiro State University - UERJ, Rio de Janeiro, Brazil \\ basilio.julio@posgraduacao.uerj.br \\ jose.ribeiro@uerj.br \\ americo.cunha@uerj.br \\ tiagoroux@uerj.br
}

\begin{abstract}
Fractional calculus is a branch of mathematics that studies the use of the fractional operators. The evolution of computing allowed its application in many areas, including control theory. Fractional operators are known to well describe memory phenomena, a favorable characteristic for control. Another benefit is that fractional-order of these operators adds more tunable parameters to the controller, allowing greater flexibility and the possibility of improving its performance. This work aims to verify the performance of an inverted cart-pendulum control system using a fractional-order integrators and discover an ideal method for optimizing systems using fractional controller. The strategy is to employ optimization methods like genetic algorithms and cross-entropy, to obtain optimal controller parameters which result in the best control performance, taking into account performance indexes related to the error, settling time and control effort. A comparison is made with the integerorder controller to verify the improvement in the use of fractional control.
\end{abstract}

Keywords: fractional controller, cross-entropy method, nonlinear dynamics, genetic algorihm

\section{Introduction}

The idea of the fractional calculus was introduced in 1695, when L'Hôpital in a letter to Leibniz questions what would be the mathematical interpretation for the notation of the derivative $d^{n} f / d x^{n}$ (created by Leibniz) if it has a non-integer order ( $n=1 / 2$, for example). The search to find this interpretation involved many mathematicians like Euler, Fourier, Laplace, among others. Its first practical application was made in 1823 by Niels Henrik Abel, to solve problem of tautochrone curve, for which the time of descent of a body abandoned on it and subject to the action of gravity is the same regardless of the starting point where the body is abandoned $[1,2]$.

During the following centuries, many pure and applied mathematicians contributed to the development of the theory of fractional calculus and many different fractional operators were proposed, including the fractional derivatives of Grünwald-Letnikov, Riemann-Liouville and Caputo [3]. 
The Riemann-Liouville fractional integral [3] of order $\alpha$, where $\alpha \in \mathbb{R}$ and $\alpha>0$, is defined in terms of a convolution type operation between the real-valued function $y(t)$ and the kernel $t^{\alpha-1}$

$$
\mathcal{I}_{a, t}^{\alpha} y(t)=\frac{1}{\Gamma(\alpha)} \int_{a}^{t}(t-\tau)^{\alpha-1} y(\tau) d \tau, \quad t>a
$$

being $\Gamma$ the Gamma function, and the Riemann-Liouville fractional derivative [3] is defined in terms of the classical derivative of order $n \in \mathbb{Z}^{+}$of this fractionalorder integral

$$
{ }_{R L} \mathcal{D}_{a, t}^{\alpha} y(t)=\frac{1}{\Gamma(n-\alpha)} \frac{d^{n}}{d x^{n}} \int_{a}^{t}(t-\tau)^{n-\alpha-1} y(\tau) d \tau,
$$

which is a global operator (not local as in classical calculus) that presents "memory", being $t>a$ and $n-1<\alpha<n$.

The development of new definitions is an active field of research in mathematics $[4,5]$, including unified definitions [6]. On the other hand, the use in applications was possible mainly due to the development of numerical methods to simulate fractional systems [7], which also allowed the use of fractional operators in engineering analysis [8] and in control theory [9], especially when dealing with delays [10] or chaotic nonlinear systems [11].

In addition to the favorable feature of fractional-order operators in providing a natural framework for describing phenomena with memory, the fractional exponent in control theory also offers a type of additional degree of freedom to tune a controller, opening up opportunities for further performance improvements in the controller project. In this context, this work proposes a LQR-based control system to stabilize an inverted cart-pendulum system and analyzes the possibility of improving the performance of the controller using fractional integrators, comparing this with the integer-order. Performance is evaluated through indexes related to the control error, the settling time of the system output and the control effort. To find the parameters that result in the best performance for the controller, including the possibility of fractional-order, and find the most efficient method to optimize systems that use fractional control, two methods of global optimization are used, the genetic algorithm [12] and the cross-entropy method [13,14]. The inverted pendulum is chosen as a reference because it is a classic control problem, a nonlinear system widely studied using integer-order controllers [15], which it has also started to be tested in the fractional control literature [16].

\section{Nonlinear dynamic system}

An inverted pendulum has its center of mass above its pivot point, so when in the vertical position is in its unstable equilibrium position, and a small disturbance can bring down the pendulum. Maintaining this equilibrium position or at a desired reference angle is done by changing the cart position. The inverted cartpendulum used in this work is shown in Figure 1 (left), where $M$ is the cart mass; 
$m$ is the inverted pendulum mass; $L$ is the distance from the center of pendulum's mass to the fixation point; $x$ is the cart's horizontal displacement; $\theta$ is the angle between the pendulum and a perpendicular axis through the cart's centroid; and $u$ is the force applied to the cart to control the system. The mass of the rod is neglected, the surface is considered to be frictionless and the mechanical joint is considered smooth.

The dynamics of the system evolve according to the nonlinear model

$$
\begin{gathered}
\left(J+m L^{2}\right) \ddot{\theta}-m g L \sin \theta=-m L \ddot{x} \cos \theta \\
(m+M) \ddot{x}+m L \ddot{\theta} \cos \theta-m L \dot{\theta}^{2} \sin \theta=u
\end{gathered}
$$

where $J$ is pendulum moment of inertia. As can be seen, the dynamics of the plant is nonlinear and a suitable controller is needed to keep the pendulum in a certain position. In this study, are considered the values of $m$ equal to $0.1 \mathrm{~kg}$, $M$ equal to $2 \mathrm{~kg}, L$ is the length of $0.5 \mathrm{~m}$ and the moment of inertia $J$ is equal to $0.006 \mathrm{~kg} \cdot \mathrm{m}^{2}$. The upper dot is an abbreviation for time-derivative.

\section{Control strategy}

\subsection{Controller design}

Figure 1 (right) shows the LQR-based controller proposed to control the inverted cart-pendulum system.
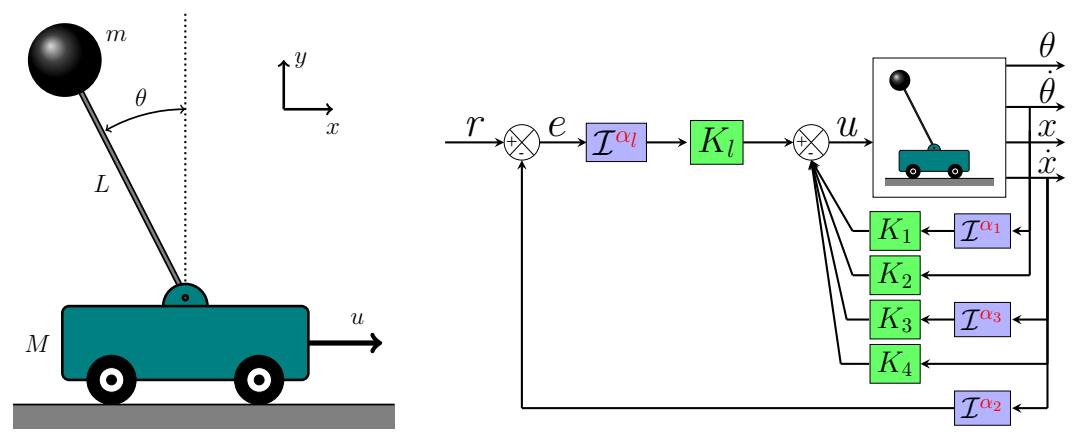

Fig. 1. Schematic illustration of the cart-pendulum system (left), and the proposed LQR-based controller (right)

In this proposed controller, $r$ is the reference input signal; $e$ is the tracking error; $u$ is the control signal; $K_{1}, K_{2}, K_{3}, K_{4}, K_{l}$ are the control gains; $\mathcal{I}^{\alpha_{1}}, \mathcal{I}^{\alpha_{2}}$, $\mathcal{I}^{\alpha_{3}}, \mathcal{I}^{\alpha_{l}}$ are the integrators, if $\alpha \in \mathbb{Z}$ (integer-order) and if $\alpha \notin \mathbb{Z}$ (fractionalorder). In this plant the feedback is done through two state variables, angular speed $\dot{\theta}$ and cart speed $\dot{x}$, chosen to be the observed states. Therefore, to obtain 
the angular position of the pendulum and the position of the car, these variables are integrated by $\mathcal{I}^{\alpha_{1}}$ and $\mathcal{I}^{\alpha_{3}}$, respectively, as defined by the fractional integral of Riemann-Liouville in Eq.(2). In addition, a feedback in the input through the integral $\mathcal{I}^{\alpha_{2}}$ and $\mathcal{I}^{\alpha_{l}}$ of the car position is performed.

To know the performance of an integer-order controller, used as a reference in the comparisons of this work, the Pole Placement method was used to obtain the controller gains $K_{i}$. This is a classic and widely used method, developed for linear systems using integer-order controllers, where the location of the poles is chosen based on the desired performance of the closed-loop system. Since this method is based on the linear system hypothesis, the inverted cart-pendulum model is linearized. Using the Matlab to calculate the gains, the following values are obtained: $K_{1}=-200.6 ; K_{2}=-50.3 ; K_{3}=-70.1 ; K_{4}=-46.8 ; K_{l}=-63.8$.

\subsection{Controller performance}

Performance indexes of control systems are indicators of the quality of the elaborated project, being possible to evaluate quantitatively the performance of different controllers. Mathematically, a performance index is a function of system states, inputs and time. In addition, performance indices can also be used as an objective function for obtaining controllers. In this work, the performance of the controllers is evaluated using the following criteria:

- Integrated square error: $I S E=\int_{0}^{t} e(t)^{2} d t$

- Settling time (ST)

- Integrated square control signal: $I S U=\int_{0}^{t} u(t)^{2} d t$

The first two indices refer to the state variables $(\theta, \dot{\theta}, x$ and $\dot{x})$. The $I S E$ index, is related to the area displaced by the car or the pendulum during the evaluated period until reaching the reference. The settling time, as the name says, is the time it takes the signal to reach the reference. The latter, the $I S U$ index, quantifies the control effort during the evaluated period.

\section{Controller optimization problem}

\subsection{LQR-based fitness function}

The objective of this work is to find the set of controller parameters that maximize the performance of the controller. Thus, the design variables chosen for the optimization problem are the gains and the order of the integrators, especially the latter that define whether the controller it will be fractional or integer.

The lower and upper bounds of the variables must be defined according to the computational capacity of the optimization method and in a way that allows the search for the global maximum, without one or more variables being close to the stipulated limit. This problem with the bounds of the variables is one of the reasons for using more than one optimization method. 
As explained in section 3.2, the performance evaluation is made through the indexes chosen here, however for the optimization process, evaluating the indexes separately would make a multi-objective optimization of high computational cost, since the number of design variables is relatively high. Therefore, it is necessary to develop an objective function that takes into account all these performance indices, including the one that assesses the control effort. Control theory has an optimized controller design technique well known in the literature called linear quadratic regulator (LQR). This technique is used in linear systems and has a quadratic cost function that includes the state variables and the control signal, in order to find the optimized gains for the control project [17].

A fitness function based on the LQR was developed mainly because it also takes into account the state variables and the control signal in the formulation

$$
\mathcal{F}=\int_{0}^{\tau}\left(w_{1} \frac{x^{2}(t)}{I S E_{x_{I}}}+w_{2} \frac{\theta^{2}(t)}{I S E_{\theta_{I}}}+w_{3} \frac{u^{2}(t)}{I S U_{u_{I}}}+w_{4} \frac{\dot{x}^{2}(t)}{I S E_{\dot{x}_{I}}}+w_{5} \frac{\dot{\theta}^{2}(t)}{I S E_{\dot{\theta}_{I}}}\right) d t
$$

where $t$ is the time variable, $\tau$ is the final evaluation time and the $w$ 's are the weights for each component of the objective function. It is worth mentioning that as the reference for the control is zero, the state variables used directly in the function quantify the error, one of the performance indices evaluated in this article. State variables are divided by the respective performance value (ISE and $I S U)$ in integer-order control, minimizing the difference in order of magnitude of each component, trying to allow the weight to be defined as much as possible by $w_{1}=w_{2}=w_{3}=0.3$ and $w_{4}=w_{5}=0.05$. Thus, the value of the fitness function for the classical controller is equal to 1 .

Therefore, to obtain a better performance $\mathcal{F}<1$ must be obtained, that is, the objective function must be minimized. To turn this minimization problem in a maximization problem, as is treated in the optimization methods applied in the article, it is considered $S=-\mathcal{F}$. The constraints are incorporated into the objective function through a penalty function

$$
P=100\left(\max \left(0, S T_{\theta}-S T_{\theta}^{*}\right)\right)^{2}+100\left(\max \left(0, S T_{x}-S T_{x}^{*}\right)\right)^{2}
$$

where $S T_{\theta}$ and $S T_{x}$ are the settling time of the angular position and the position of the car, $S T_{\theta}^{*}$ and $S T_{x}^{*}$ are the respective values in the classic controller.

\subsection{Genetic algorithms}

Genetic algorithms (GA) are heuristic search approaches applicable to a wide range of optimization problems. Invented by Holland $(1975,1992)$, this stochastic optimization and global search technique are successful methods for use in problems with difficult solution spaces, for example, if no derivatives are available and if the fitness scenario suffers from poorly conditioned parts [12].

Using the principle of genetics and natural selection, evolution is the basis of genetic algorithms, together with their main genetic operators, which are crossover, mutation and selection. Crossover operators combine the genomes of two or more solutions. The mutation operator adds randomness to the solutions. And finally, the selection operator chooses the best solutions in a population for survival [18]. 


\subsection{Cross-Entropy method}

Global search methods like genetic algorithms are quite effective in overcoming local minimums or maximums, unlike gradient-based methods, however its control parameters are not intuitive and most of the time its tunning is done through trial and error, which can lead to loss of performance and accuracy. In an attempt to overcome such difficulties and succeed in the search for the controller with the best performance, this work uses, in addition to the GA method, another global search algorithm known as the cross-entropy (CE) method. More robust and simple, it was proposed by R. Rubinstein in 1997 [13] initially for simulation of rare events, and then its effectiveness in application in optimization problems was observed $[19,20]$. The idea of the method is to transform a non-convex optimization problem into a rare event estimation problem, that can be solved by Monte Carlo sampling technique. The process consists of:

(i) sample the feasible region according to a given probability distribution;

(ii) evaluate the objective function in each of these samples;

(iii) identify the samples that produced the highest values for the objective function, this subset being defined as the elite sample set;

(iv) update the parameters of the probability distribution based on the mean and standard deviation of the elite sample set, modifying the distribution in order to try to make it as close as possible to the global optimum;

(v) repeat steps (2) to (4) as long as a stop criterion is not met.

This iterative process can be classified into two stages: sampling (i and ii) and learning (iii to v). Furthermore, this process only requires the user to define the number of samples $N_{s}$, the number of elite samples $N_{e}<N_{s}$, a tolerance of convergence tol and the maximum number of levels (iterations) $l_{\max }$ [14].

\section{$5 \quad$ Numerical experiments}

The numerical experiments presented here, consist of the execution of optimizations using GA and CE methods through numerical simulations in Matlab, where several evaluations of the objective function are performed and the ideal parameters of the controller that result in its best performance are found. For the operations of fractional integrators the FOMCON toolbox is employed [7].

The initial conditions for the numerical simulations are defined: $x_{0}=0 \mathrm{~m}$; $\dot{x}_{0}=0 \mathrm{~m} / \mathrm{s} ; \theta_{0}=10 \times \pi / 180 \mathrm{rad} ; \dot{\theta}_{0}=0 \mathrm{rad} / \mathrm{s}$. Note that the initial condition of the angular position other than 0 (zero) will force the control system to act to find stabilization. The dynamics are integrated over the time interval $[0 ; \tau]=[0 ; 10] s$.

The optimizations will be divided into two stages according to the choice of design variables to be analyzed. In the first round of optimization, it seeks to find the optimal values of $\alpha_{1}$ and $\alpha_{3}$, which results in the best performance for the controller, keeping the other parameters fixed. The strategy is to use the controller's gains, obtained by the linear Pole Placement method and consider the order of the integers $\alpha_{2}$ and $\alpha_{l}$ equal to 1 . Then, optimizations are performed with all system parameters (gains and integrators) to find the one that results in the best control performance. 


\subsection{The $\alpha_{1}$ and $\alpha_{3}$ optimization}

Initially, the GA and $\mathrm{CE}$ optimizations are made with the design variables being $\alpha_{1}$ and $\alpha_{3}$, keeping the other controller parameters fixed and equal to 1 . The results obtained in each of these optimizations are presented in Table 1.

Table 1. Optimal values of the design variables $\left(\alpha_{1}\right.$ and $\left.\alpha_{3}\right)$ with their respective performance indexes and fitness function value

\begin{tabular}{|c|c|c|c|c|c|c|}
\hline & & $\begin{array}{r}\text { integer } \\
\text { controller }\end{array}$ & $\begin{array}{r}\text { frac } \\
\text { contr }\end{array}$ & lenal $\mathrm{GA}$ & $\begin{array}{r}\text { frac } \\
\text { contr }\end{array}$ & $\begin{array}{l}\text { ional } \\
\text { ler CE }\end{array}$ \\
\hline & $\alpha_{1}$ & 1.00 & & 1.03 & & 1.03 \\
\hline & $\alpha_{2}$ & 1.00 & & 1.00 & & 1.00 \\
\hline & $\alpha_{3}$ & 1.00 & & 0.93 & & 0.93 \\
\hline & $\alpha_{l}$ & 1.00 & & 1.00 & & 1.00 \\
\hline parameters & $K_{1}$ & -200.60 & & -200.60 & & -200.60 \\
\hline & $K_{2}$ & -50.30 & & -50.30 & & -50.30 \\
\hline & $K_{3}$ & -70.10 & & -70.10 & & -70.10 \\
\hline & $K_{4}$ & -46.80 & & -46.80 & & -46.80 \\
\hline & $K_{l}$ & -63.80 & & -63.80 & & -63.80 \\
\hline & $I S E \theta$ & 0.021 & 0.017 & $-20 \%$ & 0.017 & $-20 \%$ \\
\hline & $I S E \dot{\theta}$ & 0.190 & 0.204 & $7 \%$ & 0.204 & $7 \%$ \\
\hline & $I S E x$ & 0.257 & 0.230 & $-11 \%$ & 0.230 & $-11 \%$ \\
\hline performance & $I S E \dot{x}$ & 0.660 & 0.509 & $-23 \%$ & 0.509 & $-23 \%$ \\
\hline index & $I S U$ & 23.659 & 24.412 & $3 \%$ & 24.411 & $3 \%$ \\
\hline & $S T \theta$ & 4.873 & 4.088 & $-16 \%$ & 4.088 & $-16 \%$ \\
\hline & $S T x$ & 5.073 & 5.100 & $1 \%$ & 5.100 & $1 \%$ \\
\hline fitnes & nction & 1.000 & 0.909 & $-9 \%$ & 0.909 & $-9 \%$ \\
\hline
\end{tabular}

The first highlight can be made on the efficiency of the CE method compared to GA to obtain the same result more quickly and with less computational cost. Another observation is that the use of fractional-order integrators can improve the performance of the controller, with an optimization close to $10 \%$ of the overall performance (based on the fitness function) of the controller. The ISE of the angular position had a reduction of $20 \%$ compared to the integer-order. The ISE of the angular velocity, the settling time of $x$ and the control effort were slightly higher, however, compared to the improvement obtained in the others, they are irrelevant. Some of these results are highlighted in Figure 2, with the time series of the angular position, car position and control effort.
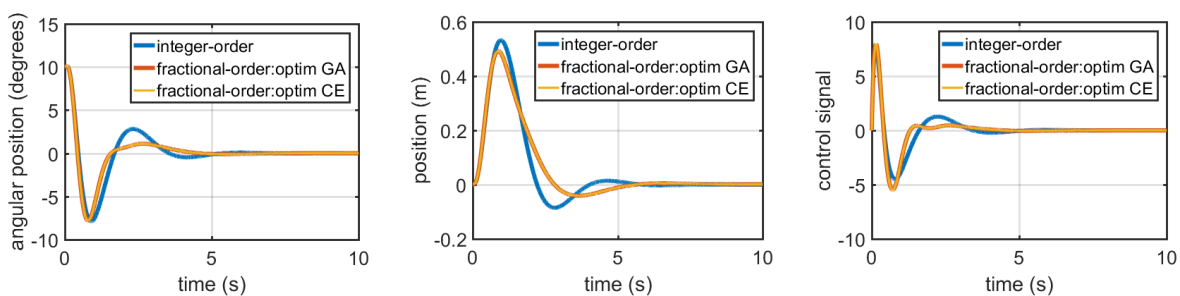

Fig. 2. Time series of the angular position (top-left), car position (top-right) and the control signal (bottom) for results firsts optimization 


\subsection{Optimization of all parameters}

Based on the results of section 5.1, the following question is asked: "if the gains are also optimized, will the integrators also have a fractional order?". To answer this question, new optimizations are developed with the GA and CE methods, now with all the parameters of the controller as design variables. The results are compared with the integer-order controller, as done previously. Table 2 shows the configuration of the methods, the bounds of all design variables and the performance of each method for optimization evaluated with all parameters.

Table 2. Bounds and performance of GA and CE algorithm for optimization evaluated

\begin{tabular}{|c|c|c|c|c|c|c|c|}
\hline method & $\alpha_{1,}$ & & $\begin{array}{c}\text { bounds } \\
K_{1}\end{array}$ & $K_{2,3}$, & & levels & $\begin{array}{l}\text { function } \\
\text { evalution }\end{array}$ \\
\hline GA & {$[0.8$} & 1.1] & {$\left[\begin{array}{lll}-250 & -150\end{array}\right]$} & {$[-90$} & $-30]$ & 327 generations & 29520 \\
\hline $\mathrm{CE}$ & {$[0.5$} & $1.5]$ & {$\left[\begin{array}{ll}-350 & -1\end{array}\right]$} & {$[-150$} & $-1]$ & 88 iterations & 4400 \\
\hline
\end{tabular}

Unlike the previous optimization, the optimizations performed with all parameters required a higher computational cost. However, as shown in Table 3, the results of the performance indices and the fitness function of the optimized parameters show that the use of fractional-order integrators together with the optimal gains can further improve the performance of the controller designed through the method of Pole Placement. The optimization methods GA and CE allowed results in general (fitness function) with $27 \%$ and $37 \%$ better than the controller used as reference, respectively.

Table 3. Controller configuration with the optimal values of the design variables (all parameters) and their respective performance indexes and fitness function value

\begin{tabular}{|c|c|c|c|c|c|c|}
\hline & & $\begin{array}{r}\text { integer } \\
\text { controller }\end{array}$ & $\begin{array}{r}\text { fra } \\
\text { contr }\end{array}$ & $\begin{array}{l}\text { ional } \\
\text { ler GA }\end{array}$ & $\begin{array}{r}\text { frac } \\
\text { contro }\end{array}$ & $\begin{array}{l}\text { ional } \\
\text { ler CE }\end{array}$ \\
\hline & & 1.00 & & 0.96 & & 0.72 \\
\hline & $\alpha_{2}$ & 1.00 & & 0.94 & & 0.79 \\
\hline & $\alpha_{3}$ & 1.00 & & 0.91 & & 1.22 \\
\hline & $\alpha_{l}$ & 1.00 & & 0.97 & & 0.80 \\
\hline parameters & $K_{1}$ & -200.60 & & -249.98 & & -205.68 \\
\hline & $K_{2}$ & -50.30 & & -60.08 & & -86.97 \\
\hline & $K_{3}$ & -70.10 & & -89.96 & & -67.54 \\
\hline & $K_{4}$ & -46.80 & & -70.35 & & -124.60 \\
\hline & $K_{l}$ & -63.80 & & -83.01 & & -65.56 \\
\hline & $I S E \theta$ & 0.021 & 0.012 & $-45 \%$ & 0.009 & $-55 \%$ \\
\hline & $I S E \dot{\theta}$ & 0.190 & 0.169 & $-11 \%$ & 0.157 & $-17 \%$ \\
\hline & $I S E x$ & 0.257 & 0.182 & $-29 \%$ & 0.136 & $-47 \%$ \\
\hline performance & $I S E \dot{x}$ & 0.660 & 0.357 & $-46 \%$ & 0.283 & $-57 \%$ \\
\hline index & $I S U u$ & 23.659 & 22.158 & $-6 \%$ & 21.497 & $-9 \%$ \\
\hline & $S T \theta$ & 4.873 & 3.952 & $-19 \%$ & 3.949 & $-19 \%$ \\
\hline & $S T x$ & 5.073 & 4.899 & $-3 \%$ & 5.100 & $1 \%$ \\
\hline fitness & unction & 1.000 & 0.730 & $-27 \%$ & 0.630 & $-37 \%$ \\
\hline
\end{tabular}

This difference in methods is linked to the better efficiency of the $\mathrm{CE}$ in relation to the operational cost (time/function evaluates). Because in GA, due 
to the CPU time and the number of variables in the problem, it was necessary to establish lower bound limits of each parameter/design variable for the method to run. This problem can be seen in the values of $K_{1}$ and $K_{3}$, which were very close to the lower bounds established for them. Another important highlight is how errors can be reduced by up to $50 \%$ with fractional integrators, yet requiring $9 \%$ less effort from the controller. The time series of the angular position, the car position and the control effort are shown in Figure 3, with the comparison of the result of each method with the integer controller.
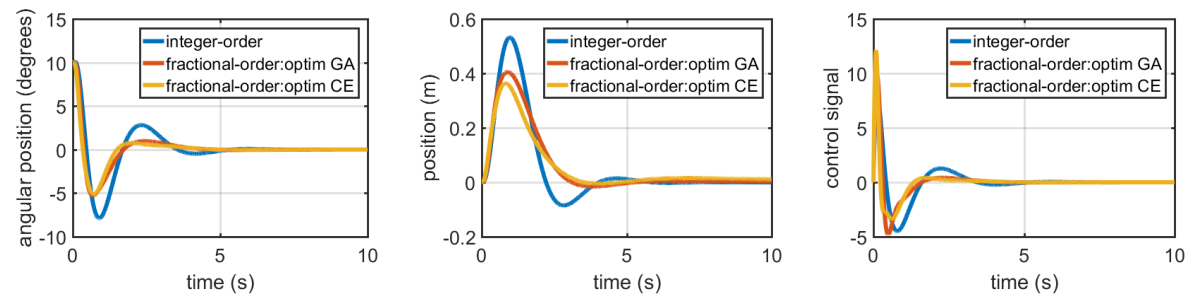

Fig. 3. Time series of the angular position (top-left), car position (top-right) and the control signal (bottom) for results optimization of the all parameters

\section{Concluding remarks}

This work used the stability problem of an inverted cart-pendulum to develop a study on the performance of fractional controllers compared to those with integer-order integrators. So there is a nonlinear non-convex optimization problem that aims to maximize the performance of the control system, taking into account the error, the settling time and the control effort. For this, two different optimization methods were used: genetic algorithms and cross entropy. The latter being faster and more robust for optimization that involves fractional-order integrators, i.e. the CE method presents better performance for problems with many variables, which is the case of systems with fractional control.

As in [9], there is an improvement in the performance of the control circuit that has fractional-order operators, but in this work for a LQR-based controller. The results show that the use of controllers with fractional integrators instead of integer-order controllers makes the control more efficient and faster, approximately $20 \%$ faster to reach the benchmark $(\theta=0 \mathrm{rad})$, with less error and requiring less effort of control. With the reduction of all control parameters, integrators and gains, the fitness function can be reduced by more than a third, allowing to find a better answer from the results of the Pole Placement method.

\section{Acknowledgements}

The authors would like to thank the financial support given to this research by the Brazilian agencies Coordenação de Aperfeiçoamento de Pessoal de Nível Superior - Brasil (CAPES) - Finance Code 001, the Conselho Nacional de Desenvolvimento Científico e Tecnológico ( $\mathrm{CNPq}$ ) and the Carlos Chagas Filho Research Foundation of Rio de Janeiro State (FAPERJ). 


\section{References}

1. Monje, C. A., Chen, Y., Vinagre, B. M., Xue, D., Feliu-Batle, V.: Fractional-order Systems and Controls - Fundamentals and Applications. Springer-Verlag (2010).

2. Ortigueira, M. D., Machado, J. A. T. : What is a fractional derivative? Journal of Computational Physics, v. 293, p. 4-13 (2015).

3. Li, C, Deng, W. : Remarks on fractional derivatives. Applied Mathematics and Computation, v. 187, n. 2, p. 777-784 (2007).

4. Caputo, M., Fabrizio, M.: A new definition of fractional derivative without singular kernel. Progr. Fract. Differ. Appl, vol. 1, n. 2, p. 1-13 (2015).

5. Zheng, Z., Zhao, W., Dai, H. : A new definition of fractional derivative. International Journal of Non-Linear Mechanics, vol. 108, p. 1-6 (2019).

6. D'Elia, M., Gulian, M., Olson, H., Karniadakis, G. : A Unified Theory of Fractional, Nonlocal, and Weighted Nonlocal Vector Calculus (2020). arxiv.org/abs/2005.07686

7. Tepljakov, A., Petlenkov, E., Belikov, J.: FOMCOM: a MATLAB toolbox for fractional-order system identification and control. International Journal of Microelectronics and Computer Science, v. 2, n. 2, p. 51-62 (2011).

8. Lin, R. M., Ng, T. Y.: Eigenvalue and eigenvector derivatives of fractional vibration systems. Mechanical Systems and Signal Processing, v. 127, p. 423-440 (2019).

9. Tepljakov, A.: Fractional-order Modeling and Control of Dynamic Systems. Springer Theses (2017).

10. Martelli, G. : Stability of PID-controlled second-order time-delay feedback systems. Automatica, v. 45, n. 11, p. 2718-2722 (2009).

11. Niu, J., Shen, Y., Yang, S., Li, S.: Analysis of Duffing oscillator with time-delayed fractional-order PID controller. International Journal of Non-Linear Mechanics, v. 92, p. 66-75 (2017).

12. Holland, J. H.: Genetic Algorithms. Scientific American, v. 267, p. 66-73 (1992).

13. Rubinstein, R. Y., Kroese, D. P.: The Cross-Entropy Method: A Unified Approach to Combinatorial Optimization, Monte-Carlo Simulation and Machine Learning. Information Science and Statistics, Springer-Verlag (2004).

14. Cunha Jr, A.: Enhancing the performance of a bistable energy harvesting device via the cross-entropy method (2020). https://hal.archives-ouvertes.fr/hal-01531845/

15. Kharola A., Patil, P., Raiwani, S., Rajput D.: A comparison study for control and stabilisation of inverted pendulum on inclined surface (IPIS) using PID and fuzzy controllers. Perspectives in Science, v. 8, p. 187-190 (2016).

16. Mousa, M. E., Ebrahim, M. A., Hassan, M. M.: Optimal fractional-order proportional-integral - differential controller for inverted pendulum with reduced order linear quadratic regulator. In: Fractional-order Control and Synchronization of Chaotic Systems, v. 688, p. 225-252, Springer, Cham (2017).

17. Anderson, B. D. O., Moore, J. B.: Optimal Control: Linear Quadratic Methods. Prentice-Hall, Inc. USA (1990).

18. Kramer, O.: Genetic Algorithm Essentials, Oldenburg, Germany: Springer (2017).

19. Kroese, D. P., Rubinstein, R. Y., Cohen, I., Porotsky, S., Taimre, T.: CrossEntropy Method. In: Encyclopedia of Operations Research and Management Science. Springer (2013). https://doi.org/10.1007/978-1-4419-1153-7_131

20. Rubinstein, R. Y., Kroese, D. P.: Simulation and the Monte Carlo Method. Wiley Series in Probability and Statistics, 3rd ed., Wiley (2016). 